\title{
Contact Normal Submanifolds and Contact Generic Normal Submanifolds in Kenmotsu Manifolds
}

\author{
MINORU KOBAYASHI \\ Dedicated to Dr. Shisanji Hokari on bis 80tb birthday
}

\begin{abstract}
We study contact normal submanifolds and contact generic normal submanifolds in Kenmotsu manifolds and in Kenmotsu space forms. Submanifolds mentioned above with certain conditions in Kenmotsu space forms are shown that they are CR-manifolds, spaces of constant curvature, locally symmetric and Einsteinnian. Also, the non-existence of totally umbilicial submanifolds in a Kenmotsu space form -1 is proven under a certain condition.
\end{abstract}

\section{INTRODUCTION}

The differential geometry of CR or semi-invariant submanifolds in Riemannian manifolds such as Kaehlerian (including Hermitian), Sasakian, product Riemannian and locally product Riemannian manifolds have been studied by many geometers (concerning the above, see [1] and [10]) Also, Quaternion CR sub-manifolds of quaternion manifolds and QR-submanifolds of quaternion Kaehlerian manifolds have been studied ([1]). We studied symmetric twofold CR-submanifolds in a Euclidean space $\mathbf{R}^{4 n}$ which is a special quaternion Kaehlerian manifold with global Kaehlerian quaternion structure ([6]). Also we studied semi-invariant sub-manifolds in $\mathrm{K}$-manifolds,

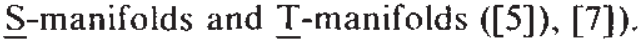

On the other hand, Kenmotsu studied a class of almost contact Riemannian manifolds ([3]). The almost contact Riemannian manifolds which belong to the class mentioned above are nowadays called Kenmotsu manifolds ([2]), [4]), [8]). 
Examples of them were given in , [3] and [8]. which we state in the following: let $\tilde{N}$ be a Kaehler manifold and $f: \mathrm{R} \rightarrow \mathrm{R}$ be a function defined by $f(t)=c e^{\prime}$. where $c^{\prime} \in \mathrm{R}: \cdot>0$. . Then the warped product $\widetilde{M}=\mathrm{R} \cdot{ }^{\prime} \widetilde{N}$ is a Kenmotsu manifold.

We studied submanifolds in Kenmotsu manifolds whose structure vector field $\xi$ is tangent to the submanifolds ([4]). Papaghiiuc studied submanifolds in Kenmotsu manifolds whose structure vector field $\xi$ is tangent to the submanifolds and normal to them extensively ([8]). Thus, it is natural to study them further.

The purpose of the present paper is to study, what we call, contact normal submanifolds and contact generic normal submanifolds in Kenmotsu manifolds and mainly those in Kenmotsu space forms (the notion of Kenmotsu space forms is the one which is analogous to Sasakian spacc forms in Sasakian geometry and the notion of contact normal submanifolds corresponds to that of $\xi^{-}$-submanifolds in [8]). One of the typical examples of Kenmotsu space form $c, \widetilde{M}(c)$, is the hyperbolic space of constant curvature -1 (when $c=-1)$.

In Section 1, we survey the fundamental "properties of Kenmotsu manifolds, give the fundamental formulas of submanifolds when the ambient manifolds are Kenmotsu space forms and definitions of contact normal and contact generic normal submanifolds in Kenmotsu manifolds and finally state the result which was obtained in [8] for later use.

In Section 2, we treat totally umbilical contact normal submanifolds and point out that they are extrinsic,spheres (totally umbilical submanifolds with parallel mean curvature vector) if $\mathrm{dim}$. $\mathrm{D}^{\perp}>\mathrm{I}$ (for the definition of $\mathrm{D}^{\perp}$, see Section 1). Also we prove the non-existence of totally umbilical contact normal submanifolds with dim. D $1 \cdot>1$ immersed in $\widetilde{M},(c)$ with $c \neq-1$ and that the submanifolds with the same conditions mentioned above are CRmanifolds (for CR-manifolds, see [1]).

In Section 3, we treat contact normal submanifolds of codimension 2 mainly in $\widetilde{M}(c)$. We prove that if $M$ is a contact normal submanifold of codimension 2 in $\widetilde{M}(c)$ and if either the second fundamental formi is parallel or the mean curvature vector field is parallel, then $c=-1$ or $M$ is antiinvariant and that $M$ is locally symmetric. And we also prove that if $M$ is a totally umbilical contact normal submanifold in $\widetilde{M}(c)$, then $M$ is a space of constant curvature if $M$ is proper (the assumption that $\operatorname{dim} . \mathrm{D}^{\perp}>1$ is excluded, cf. [8]).

In the final Section 4 , we treat contact generic normal submanifolds and contact generic normal products mainly in $\widetilde{M}(c)$. Several results concerning contact generic normal products'such as the integrability conditions of the 
distributions induced on the submanifold. properties of leaves of them and properties of the canonically induced structures on $M$ etc are obtained. At the end of this section, we treat hypersurfaces in Kenmotsu manifolds whose structure vector field is normal to the hypersurfaces. Main results in this case are that (1) if a hypersurface $M$ normal to the structure vector field $\xi$ is in $\widetilde{M}(c)$, then $M$ is locally symmetric, (2) $M$ is flat if and only if $c=-1$ and (3) if $M$ is in $\widetilde{M}(c)$ with $c \neq-1$, then $M$ is Einsteinnian.

\section{PRELIMINARES}

Let $\widetilde{M}=\widetilde{M}^{2 m+1}$ be a $(2 m+1)$-dimensional almost contact metric manifold with structure $(\phi, \xi, \eta,<,>)$. where $\phi$ is a $(1,1)$ type tensor fields, $\xi$ is a vector field, $\eta$ is a 1 -form and $\langle$, $>$ is the associated Riemannian metric on $M$. Then, by definition ([9]), we have

$$
\begin{aligned}
& \phi^{2}=-I+\eta \otimes \xi, \eta(\xi)=1, \phi \xi=0, \eta \circ \phi=0, \\
& <\phi \widetilde{X}, \phi \widetilde{Y}>=\langle\widetilde{X}, \widetilde{Y}>-\eta(\widetilde{X}) \eta(\widetilde{Y}), \eta(\widetilde{X})=\langle\widetilde{X}, \xi>,
\end{aligned}
$$

where 1 is the identy tensor field and $\widetilde{X}, \widetilde{Y}$ are vector fields in $\widetilde{M}$. Let $M$ be an n-dimensional isometrically immersed submanifold in $\tilde{M}$. Let $T(M)$ and $T(M)^{\perp}$ be the tangent bundle and normal bundle of $M$ respectively.

Definition 1. $M$ is called to be a contact normal submanifold in $\tilde{M}$ if the structure vector field $\xi$ is normal to $M$ and if there exists a differentiable distribution $D$ on $M$ such that

$$
T(M)=\underline{D} \oplus \underline{D}^{\perp}, \quad \phi \underline{D}=\underline{D}, \quad \phi \underline{D}-\subset T(M)^{\perp},
$$

where $D^{\perp}$ is the complementary distribution of $D$ in $T(M)$.

Definition 2. Mis called to be a contact generic normal submanifold in $\tilde{M}$ if the structure vector field $\xi$ is normal to $M$ and

$$
\phi\left(T(M)^{\perp}\right) \subset T(M) .
$$

holds.

Definition 3. A contact normal submanifold $M$ is called $\phi$-invariant (resp. anti-invariant) if $\underline{D}^{\perp}=0$ (resp. $\left.\underline{D}=0\right)$. 
We call a contact normal submanifold $M$ is proper if it neither $\phi$-invariant $\left(\underline{D}^{\perp}=0\right)$ nor anti-invariant $(\underline{D}=0)$.

Now, we recall that $\widetilde{M}$ is called a Kenmotsu manifold if

$$
\left(\widetilde{\nabla}_{\tilde{r} \phi}\right) \widetilde{Y}=<\phi \widetilde{X}, \widetilde{Y}>\xi-\eta(\widetilde{Y}) \phi \tilde{X}, \quad \widetilde{\nabla}_{\tilde{x} \xi}=\tilde{X}-\eta(\tilde{X}) \xi,
$$

where $\widehat{\nabla}$ is the covariant differentiation on $\tilde{M}$.

Now, the formulas of Gauss and Weingarten are given respectively by

$$
\begin{aligned}
& \widetilde{\nabla}_{X} Y=\nabla_{X} Y+B(X, Y), \\
& \widetilde{\nabla}_{X} N=-A_{N} X+\nabla_{Y}^{\prime} N,
\end{aligned}
$$

where $\nabla$ is the Riemannian connection determined by the induced metric $<,>$ on the submanifold $M, \nabla^{\perp}$ is the metric connection on $T(M)^{\perp}$ and both $B$ and $A$ are called the second fundamental tensors (or forms) satisfying $\langle B(X, Y), N\rangle=\langle A, X, Y\rangle, X, Y$ being tangent vector fields to $M$ and $N$ being a normal vector field to $M$.

The mean curvature vector field $H$ is defined by

(1.8) $H=\frac{1}{n}$ trace $B$.

$M$ is called minimal if $H=0$, totally umbilical if $B(X, Y)=\langle X, Y\rangle H$ and totally geodesic if $B=0$ identically. And, the mean curvature vector field $H$ is called parallel if $\nabla_{X}^{\frac{1}{X}} H=0$. Let $\widetilde{\mathrm{R}}$ (resp. R) be the curvature tensor of $\widetilde{M}$ (resp. $M$ ). Then the equations of Gauss, Weingarten and Ricci are given respectively by

$$
\begin{aligned}
& <\widetilde{R}(X, Y) Z, W\rangle=\langle R(X, Y) Z, W>- \\
& \langle B(X, W), B(Y, Z)>+<B(Y, W), B(X, Z)>
\end{aligned}
$$

$$
\begin{aligned}
& (\widetilde{R}(X, Y) Z)^{\perp}=\left(\bar{\nabla}_{X} B\right)(Y, Z)-\left(\bar{\nabla}_{Y} B\right)(X, Z), \\
& \left\langle\widetilde{R}(X, Y) N, N^{\prime}\right\rangle=\left\langle R^{\perp}(X, Y) N, N^{\prime}\right\rangle-\left\langle\left[A_{N}, A_{N}\right] X, Y\right\rangle
\end{aligned}
$$

where $(R(X, Y) Z)^{\prime}$ in $(1.10)$ is the normal component of $\widetilde{R}(X, Y) Z$, and where

$$
\begin{aligned}
& \left(\bar{\nabla}_{X} B\right)(Y, Z)=\nabla_{X}^{\perp} B(Y, Z)-B\left(\nabla_{X} Y, Z\right)-B\left(Y, \nabla_{X} Z\right), \\
& R^{\perp}(X, Y)=\nabla_{\perp}^{\perp} \nabla_{Y}^{\perp}-\nabla_{Y}^{\perp} \nabla_{X}^{\perp}-\nabla_{[X, Y]}^{\perp}, \\
& {\left[A_{N}, A_{N}\right]=A_{N} A_{N^{N}}-A_{N^{\prime}} A_{N},}
\end{aligned}
$$


$N$ and $N^{\prime}$ being normal vector fields to $M$. The second fundamental tensor $B$ is called parallel if $\bar{\nabla}_{X} B=0$ identically. And, if $R^{\downarrow}=0$ identically, then we say that the normal connection of $M$ is flat (or rrivial).

Now, we recall that if $\widetilde{M}$ is a Kenmotsu space form c, then the curvature tensor of $\widetilde{M}$ has the form

(1.15) $4 \widetilde{R}(X, Y) Z=(c-3)(<\widetilde{Y}, \widetilde{Z}>\widetilde{X}-<\widetilde{X}, \widetilde{Z}>\widetilde{Y})+$ $+(c+1)\{\eta(\widetilde{X}) \eta(\widetilde{Z}) \widetilde{Y}-\eta(\widetilde{Y}) \eta(\widetilde{Z}) \widetilde{X}$ $+\eta(\widetilde{Y})<\widetilde{X}, \widetilde{Z}>\xi-\eta(\widetilde{X})<\widetilde{Y}, \widetilde{Z}>\xi+<\widetilde{X}, \phi \widetilde{Z}>\phi \widetilde{Y}-$ $-<\widetilde{Y}, \phi \widetilde{Z}>\phi \widetilde{X}+2<\widetilde{X}, \phi \widetilde{Y}>\phi \widetilde{Z}\}$.

We denote a Kenmotsu space form $c$ by $\widetilde{M}(c)$. Then, if the ambient manifold is a Kenmotsu space form $\tilde{M}(c)$ and $M$ is a contact normal submanifold in $\widetilde{M}(c)$, from (1.9), (1.10), (1.11) and (1.15) we have

$$
\begin{aligned}
& <R\left(X, Y>Z, W>=\frac{1}{4}(c-3)(<Y, Z><X, W>-<X, Z><Y, W>)\right. \\
& +\frac{1}{4}(c+1)(<X, \phi Z><\phi Y, W>-<Y, \phi Z><\phi X, W>+ \\
& +2<X, \phi Y><\phi Z, W>) \\
& +<B(X, W), B(Y, Z)>-<B(Y, W), B(X, Z)>
\end{aligned}
$$

$$
\begin{aligned}
& \left(\bar{\nabla}_{X} B\right)(Y, Z)-\left(\bar{\nabla}_{Y} B\right)(X, Z)=\frac{1}{4}(c+1)\left\{<X, \phi Z>(\phi)^{\perp-}\right. \\
& \left.-<Y, \phi Z>(\phi X)^{\perp}+2<X, \phi Y>(\phi Z)^{\perp}\right\}
\end{aligned}
$$

$$
\begin{aligned}
& <R^{\perp}(X, Y) N^{\prime} N^{\prime}>=\frac{1}{4}(c+1)\left(<X, \phi N><\phi Y, N^{\prime}>-\right. \\
& \left.-<Y, \phi N><\phi X, N^{\prime}>\right) \\
& +<\left[A_{N^{\prime}}, A_{N}\right] X, Y>
\end{aligned}
$$

where $(\phi Y)^{\perp}$ in (1.17) denotes the normal component of $\phi Y$, etc.

Now, we recall that $M$ is called a $C R$-manifold if there exist a differentiable distribution $\underline{\mathrm{D}}$ on $M$ and an endomorphism $J$ such that $J^{2}=-1$ on $\underline{\mathrm{D}},[J, J](X, Y)=0$ and $\left.[J X, J Y]-[X, Y] \in \underline{\mathrm{D}}\right)(X, Y \in \underline{\mathrm{D}})[J, J]$ being the Nijentuis tensor of $J$ (see [l]).

The following result is used later. 
Proposition A([8]). Let $M$ be a contact normal submanifold in a Kenmotsu manifold. Then we have mal,

(a) $\mathrm{A}_{\xi} X=-X$, (b) $\nabla_{\mathrm{i}} \xi=0$, (c) $\langle\mathrm{H}, \xi\rangle=-1$, (d) $M$ is never mini-

(e) $M$ is totally umbilical if and only if $B(X, Y)=-\langle X, Y\rangle$ provided that dim. Di>l.

\section{TOTAILY UMBILICAL CONTACT NORMAL SUBMANIFOLDS AND EXTRINSIC SPHERES IN KENMOTSU MANIFOLDS}

Let $M$ be a proper contact normal submanifold in a Kenmotsu manifold and let $N_{1}=\xi, N_{2} \ldots, N_{r}(r=2 m+1-n)$ be the orthonormal vector fields normal to $M$. Then the second fundamental tensor $B$ is expressed by

$$
B(X, Y)=\sum_{i=1}^{r}<A_{i} X, Y>N_{i}=-<X, Y>\xi+\sum_{j=2}^{r}<A_{j} X, Y>N_{j}
$$

where we put $A_{i}=A_{N_{i}}$. Then the mean curvature vector field $H$ is given by

$$
H=\frac{1}{n} \operatorname{trace} B=-\xi+\sum_{j=2}^{r}\left(\operatorname{trace} A_{j}\right) N_{j} \text {. }
$$

And, the length $\|H\|$ of $H$ is given by

$$
\|H\|^{2}=1+\sum_{j=2}^{r}\left(\operatorname{trace} A_{j}\right)^{2}
$$

Now, from Proposition $A,(2.1)$ and (2.3) we immediately have

Proposition 2.1 Let $M$ be a proper contact normal submanifold with dim. $D^{\perp} \geq 1$ in a Kenmotsu manifold $\widetilde{M}$. Then $M$ is rotally umbilical in $\widetilde{M}$ if and only if

$$
A_{j}=\left(\operatorname{trace} A_{j}\right) \text { I }(j=2, \ldots, r) .
$$

Proposition 2.2. A totaly umbilical proper contact normal submanifold in a Kenmotsu manifold is necessarily an extrinsic sphere provided that $\operatorname{dim} . \underline{D}>1$.

Theorem 2.3. There exist no proper totally umbilical contact normal submanifolds in a Kenmotsu space form $\widetilde{M}(c)$ with $c \neq-I$ if dim. $D^{\perp}>l$. 
Proof. If $M$ is totally umbilical with dim. $\mathrm{D}^{\perp}>1$, we have $B(X, Y)=$ $=\langle X, Y\rangle H=-\langle X, Y\rangle \xi$ by Proposition $A$. Also since $\nabla_{X} \xi=0$ by Propositions $A$, we have $(\bar{\nabla}, B)(Y, Z)=0$ and hence from (1.17) with $Z=X$, we have

$$
0=\frac{3}{4}(c+1)<X, \phi Y>(\phi X)^{\perp},
$$

from which we must have $c=-1$ because $M$ is neither $\phi$-invariant nor antiinvariant, completing the proof.

Lemma 2.4. Let $M$ be a contact normal $\phi$-invariant submanifold of codimension $r>2$ in a Kenmorsu manifold. Then

(a) $\nabla_{X} \phi Y=\phi \nabla_{X} Y\left(\Longleftrightarrow \nabla_{X} \phi=0\right)$,

(b) $B(X, \phi Y)-\phi B(X, Y)=\langle\phi X, Y>\xi$

holds.

Proof. Using the first equation of (1.4), we have

$$
\left(\widetilde{\nabla}_{X} \phi\right) Y=\langle\phi X, Y>\xi-\eta(Y) \phi X=\langle\phi X, Y>\xi .
$$

On the other hand, we have

$$
\left(\widetilde{\nabla}_{X} \phi\right) Y=\widetilde{\nabla}_{X} \phi Y-\phi \widetilde{\nabla}_{X} Y=\nabla_{X} \phi Y+B(X, \phi Y)-\phi \nabla_{X} Y-\phi B(X, Y)
$$

Since $M$ is $\phi$-invariant, we see that $\phi B(X, Y) \in T(M)^{\perp}$. Thus, comparing the above two equations and taking the tangent and normal parts respectively, we have our assertion, completing the proof.

Theorem 2.5. Let $M$ be a contact normal totally umbilical submanifold with dim. $D^{\perp}>I$ in a Kenmotsu manifold. Then $M$ is a $C R$-manifold with CR-structure $(\phi, D)$.

Proof. This follows from Lemma 2.4 and the definition of CRmanifolds.

Remark. The assumption that the codimension $r>2$ in Lemma 2.4 is essential because there exist no contact normal $\phi$-invarinat submanifolds of codimension 2 in Kenmotsu manifolds (see Theorem 3.5 in the next section). 


\section{CONTACT NORMAL SUBMANIFOLDS OF CODIMENSION 2 IN KENMOTSU MANIFOLDS}

Let $M$ be a submanifold of codimension 2 in a Kenmotsu manifold $\widetilde{M}$. Since $M$ is of codimension 2, we may put

$$
B(X, Y)=\left\langle A_{N}, X, Y>N+\left\langle A_{,}, X, Y>N^{\prime}\right.\right.
$$

where $N$ and $N^{\prime}$ are orthonormal vector fields normal to $M$.

From (1.16)-(1.18) and (3.1), the equations of Gauss, Codazzi and Ricci are given respectively by

$(3,2)<\widetilde{R}(X, Y) Z, W>=<R(X, Y) Z, W>-<B(X, W), B(Y, Z)>+$

$+<B(Y, W), B(X, Z)>$

$=\left\langle R(X, Y) Z, W>-<A_{N} X, W><A_{A} Y, Z>-\right.$

$-\left\langle A_{N}, X, W\right\rangle\left\langle A_{N}, Y, Z\right\rangle$

$\left.+<A_{N^{\prime}} Y, W\right\rangle\left\langle A_{N^{\prime}} X, Z\right\rangle+\left\langle A_{N^{*}} Y, W\right\rangle\left\langle A_{N^{*}} X, Z\right\rangle$,

(3.3) $(\widetilde{R}(X, Y) Z)^{\mu}=\left(\bar{\nabla}_{X} B\right)(Y, Z)-\left(\bar{\nabla}_{Y} B\right)(X, Z)$

$=\left\langle A_{N} Y, Z>\nabla_{Y}^{\frac{1}{Y}} N-\left\langle A_{N} X, Z>\nabla_{Y}^{\frac{1}{1}} N+\left\langle A_{N^{\prime}} Y, Z>\nabla_{\frac{1}{1}}^{\prime} N^{\prime}-\right.\right.\right.$ $-<A_{N}, X, Z>\nabla_{!}^{\lrcorner} N^{\prime}$

$+\left(<\left(\nabla_{X^{\prime}} A\right)_{N} Y, Z>-<\left(\nabla_{Y} A\right)_{N} X, Z>+<A_{\nabla_{Y}^{1} Y} Y, Z>-\right.$

$\left.-<A_{\nabla+}^{\perp} X, Z>\right) N$

$+\left(\left\langle\left(\nabla_{,} A\right)_{N} Y, Z>-<\left(\nabla_{Y} A\right)_{N}, X, Z>+<A_{\nabla_{Y}^{\perp}, Y, Z}, Z-\right.\right.$

$-\left\langle A_{\nabla}^{ \pm} \cdot X, Z>N^{\prime}\right.$

(3.4) $<\widetilde{R}(X, Y) N, N^{\prime}>=<R^{\perp}(X, Y) N, N^{\prime}>-<\left[A_{N^{\prime}}, A_{N^{\prime}}\right] X, Y>$.

where $\left(\nabla_{X} A\right)_{N} Y=\nabla_{X} A_{N} Y-A_{\nabla^{\prime}} Y-A_{N} \nabla_{X} Y$. it is seen that $\bar{\nabla}_{X} B=0 \Longleftrightarrow$ $\left(\nabla_{X} A\right)_{N}=0$

Now, if the ambient Kenmotsu manifold $\widetilde{M}$ is a Kenmotsu space form $c$, then from $(1.16)-(1.18)$ and $(3.1)-(3.4)$ we have

(3.5) $<R(X, Y) Z, W>=\frac{1}{4}(c-3)(<Y, Z><X, W>-$

$-<X, Z><Y, Z><Y, W>)$ 
$+\frac{1}{4}(c+1)(<X, \phi Z><\phi Y, W>-<Y, \phi Z><\phi X, W>+$

$+2<X, \phi Y>\langle\phi Z, W\rangle)$

$+<A_{N} X, W><A_{N} Y, Z>+<A_{N^{\prime}} X, W><A_{N}, Y, Z>-$

$-<A_{N} Y, W>\left\langle A_{N} X, Z>\right.$

$-\left\langle A_{N^{\prime}} Y, W\right\rangle\left\langle A_{N^{*}} X, Z\right\rangle$

(3.6)

$<A_{N^{\prime}} Y, Z>\nabla_{\vec{X}}^{+} N-<A_{,} X, Z>\nabla_{\vec{y}}^{\perp} N+<A_{N^{\prime}} Y, Z>\nabla_{\bar{x}^{\prime}} N^{\prime}-$

$-<A_{N^{\prime}} X, Z>\nabla_{\frac{1}{Y}}^{\perp} N^{\prime}$

$+\left(\left\langle\left(\nabla_{X} A\right)_{N} Y, Z>-<\left(\nabla_{Y} A\right)_{N} X, Z>+<A_{\nabla_{Y}}, Y, Z>-\right.\right.$

$\left\langle A_{\nabla_{1}^{1}}, X, Z>\right) N$

$+\left(\left\langle\left(\nabla_{X} A\right)_{N}, Y, Z\right\rangle-<\left(\nabla_{Y} A\right)_{N}, X, Z\right\rangle+<A_{\nabla_{i} \cdot} Y, Z>-$

$\left\langle A_{\nabla+1} X, Z>\right) N^{\prime}$

$=\frac{1}{4}(c+1)\left(<X, \phi Z>(\phi Y)^{\perp-}<Y, \phi Z>(\phi X)^{+}+2<X, \phi Y>(\phi Z)^{+}\right.$,

(3.7) $\left\langle R^{\perp}(X, Y) N, N^{\prime}\right\rangle=\frac{1}{4}(c+1)\left(<X, \phi N><\phi Y, N^{\prime}>-\right.$

$\left.-<Y, \phi N\rangle\left\langle\phi X, N^{\prime}\right\rangle\right)+<\left[A_{N}, A_{N^{\prime}}\right] X, Y>$,

Hereafter we take $\xi$ and $\zeta$ as unit orthonormal normal vector fields to $M$. We first note that

(3.8) $\left(\nabla_{X} A\right)_{\xi} Y=\nabla_{X} A_{\xi} Y-A_{\nabla_{k}^{1} \varepsilon} Y-A_{\xi} \nabla_{X} Y=-\nabla_{A^{\prime}} Y+\nabla_{X} Y=0$

by virtue of Proposition A. Now, differentiating $\langle\xi, \zeta\rangle=0$ covariantly, we have $\left\langle\xi, \nabla_{*}^{\perp} \zeta\right\rangle=0$ because of $\nabla_{\dot{H}}^{\perp} \xi=0$. Since we easily see that $\left\langle\nabla_{\vec{r}} \zeta, \zeta\right\rangle=0$, we have

Lemma 3.1. $\zeta$ is parallel in the normal bundle $T(M)$-.

Hereafter we assume that $M$ is a contact normal submanifold of codimension 2 with dim. $\underline{\mathrm{D}}^{\perp}>1$ in a Kenmotsu manifold and put $A=A_{\zeta}$. Then the second fundamental form $B$ is expressed by

$$
B(X, Y)=\left\langle A_{\xi} X, Y>\xi+<A_{\zeta} X, Y>=-<X, Y>\xi+<A X, Y>\zeta .\right.
$$

Then, taking account of $\nabla_{x}^{\perp} \xi=\nabla_{x}^{\perp} \zeta=0$ and using (3.9), we have $\left(\bar{\nabla}_{Z} B\right)(X, Y)=\nabla_{Z}^{\perp} B(X, Y)-B\left(\nabla_{Z} X, Y\right)-B\left(X, \nabla_{Z} Y\right)$ 


$$
\begin{aligned}
= & -\left(\left\langle\nabla_{Z} X, Y>+<X, \nabla_{Z} Y>\right) \xi+\left(<\left(\nabla_{Z} A\right) X, Y>+\right.\right. \\
& \left.<A \nabla_{Z} X, Y>\xi+<A X, \nabla_{Y} Y>\right) \zeta \\
+ & <\nabla_{Z} X, Y>\xi-<A \nabla_{X} X, Y>\xi+<X, \nabla_{Y} Y>\xi- \\
- & <A X, \nabla_{Z} Y>\zeta=<\left(\nabla_{Y} A\right) X, Y>\zeta .
\end{aligned}
$$

Therefore, by (3.8) and the above identity, we have

Lemma 3.2. The second fundamental form $B$ is parallel if and only if $\nabla_{Z} A=0$

Now, the mean curvature vector field $H$ is given by

$$
\begin{aligned}
H & =\frac{1}{2 m-1} \operatorname{trace} B=\frac{1}{2 m-1} \sum_{i=1}^{2 m-1}\left\{-<e_{i}, e_{i}>\xi+<A e_{i}, e_{i}>\zeta\right\} \\
& =-\xi+\frac{1}{2 m-1}(\operatorname{trace} A) \zeta,
\end{aligned}
$$

where $\left\{e_{i}\right\}$ is an orthonormal base of $T(M)$. Then from (3.10) we have

(3.11) $\|H\|^{2}=\left\langle H, H>=1+\frac{1}{(2 m-1)^{2}}(\text { trace } A)^{2}\right.$.

Therefore, from (3.10) and (3.11), we have

Lemma 3.3. The following conditions are mutually equivalent:
(a) $\operatorname{trace} A=0$,
(b) $H=-\xi$,
(c) $\|H\|=1$.

Remark 1. If $M$ is totally umbilical then we have (3.12).

Now, differentiating (3.10) covariantly, taking account of $\nabla_{A}^{\perp} \xi=\nabla_{x}^{\perp} \zeta=0$ and Proposition $A$, we have
(a) $A_{n} X=X+\frac{1}{2 m-1}(\operatorname{trace} A) A X$,
(b) $\nabla_{X} H=\frac{1}{2 m-1}\left(\operatorname{trace} \nabla_{X} A\right) \zeta$

Therefore, from I.emma 3.2 and (3.13), we have 
Lemma 3.4. The following conditions are mutually equivalent:
(a) $B$ is parallel,
(b) $H$ is parallel.
(c) $\nabla_{X} A=0$.

We are now in a position to prove the non-existence of contact $\phi$-invariant normal submanifolds in Kenmotsu manifolds. We have

Theorem 3.5. There exist no contact $\phi$-invariant normal submanifolds of codimension 2 in a Kenmotsu manifold.

Proof. It is clear that $\phi \zeta \in T(M)$ because of $\langle\phi \zeta, \xi\rangle=\langle\phi \zeta, \zeta\rangle=0$. Assume that $M$ is a contact $\phi$-invariant normal submanifold. Then, for $X \in T(M)$, we have $\langle\phi \zeta, X\rangle=-\langle\zeta, \phi X\rangle=0$, which implies $\phi \zeta=0$. Hence we have $\zeta=0$, which is a contradiction.

Hereafter we confine our submanifold $M$ to be a contact normal submanifold of codimension 2 in a Kenmotsu space form $\tilde{M}(c)$. Then, putting $N=\xi$ and $N^{\prime}=\zeta$ in (3.5) $-(3.7)$, we have

(3.15) $\left\langle R(X, Y) Z, W>=\frac{1}{4}(c+1)\{<Y, Z><X, W>-<X, Z><Y, W>\right.$ )

$$
\begin{aligned}
& +<X, \phi Z\rangle\langle\phi \phi Y, W\rangle-\langle Y, \phi Z\rangle\langle\phi X, W\rangle+ \\
& +2<X, \phi Y><\phi Z, W>\} \\
& +<A X, W\rangle\langle A Y, Z>-\langle A Y, W\rangle\langle A X, Z\rangle
\end{aligned}
$$

$$
\begin{aligned}
(< & \left.\left(\nabla_{X} A\right) Y, Z>-<\left(\nabla_{Y} A\right) X, Z>\right) \zeta \\
= & \frac{1}{4}(c+1)\left(<X, \phi Z>(\phi Y)^{\perp}-<Y, \phi Z>(\phi X)^{\perp}+\right. \\
& +2<X, \phi Y>(\phi Z)^{\perp}
\end{aligned}
$$

(3.17) $\left\langle R^{+}(X, Y) \xi, \zeta>=0\right.$,

because of $\nabla_{X}^{\perp} \xi=\nabla_{X}^{1} \zeta=0, A_{\xi} X=-X$ and $\left[A_{\xi}, A_{\zeta}\right]=0$.

Remark. We see from (3.17) that the normal connection of $M$ is flat.

Also, we have

Theorem 3.6. Let $M$ be a contact normal submanifold of codimension 2. in a Kenmotsu space form $\widetilde{M}(c)$. If the second fundamental tensor $B$ is 
parallel or the mean curvature vector field $H$ is parallel or $\nabla_{\mathrm{X}} \mathrm{A}=0$, then $c=-1$ or $M$ is an anti-invariant submanifold.

Proof. By assumption and Lemma 3.4, the left handside of (3.16) vanishes. Since $\phi \zeta$ is a tangent vector field to $M$, putting $Z=\phi \zeta$ in the right handside of (3.16) we have

$$
\begin{aligned}
\text { R.H.S. of (3.16) }= & \frac{1}{4}(c+1)\left(<X, \phi^{2} \zeta>(\phi Y)^{\perp-}<Y, \phi^{2} \zeta>(\phi X)^{\perp}+\right. \\
& \left.+2<X, \phi Y>\left(\phi^{2} \zeta\right)^{\perp}\right) \\
= & \frac{1}{4}(c+1)\left\{-<X, \zeta>(\phi Y)^{\perp}+<Y, \zeta>(\phi X)^{\perp}-\right. \\
& -2<X, \phi Y>\zeta\} \\
= & -\frac{1}{2}(c+1)<X, \phi Y>\zeta .
\end{aligned}
$$

from which we see that $c=-1$ or $M$ is an anti-invariant submanifold, completing the proof.

Theorem 3.7. Let $M$ be a contact normal submanifold of codimension 2 in a Kenmotsu space form $\widetilde{M}(c)$. If the second fundamental tensor $B$ is parallel or the mean curvature vector field $H$ is parallel or $\nabla_{X} A=0$, then $M$ is locally symmetric.

Proof. By assumption and Theorem 3.7, from (3.15) if $c=-1$, we have

$$
R(X, Y) Z=<A Y, Z>A X-<A X, Z>A Y \text {. }
$$

Thus, taking account of $\nabla^{\perp} \zeta=0$, we have

$$
\begin{aligned}
& \left(\nabla_{U} R\right)(X, Y) Z=\nabla_{U} R(X, Y) Z-R\left(\nabla_{U} X, Y\right) Z-R\left(X, \nabla_{U} Y\right) Z- \\
& \quad-R\left(X, Y \nabla_{U} Z\right. \\
& =\nabla_{U}(<A Y, Z>A X-<A X, Z>A Y)-<A Y, Z>A \nabla_{U} X+ \\
& \quad+<A \nabla_{U} X, Z>A Y \\
& -<A \nabla_{U} Y, Z>A X+<A X, Z>A \nabla_{U} Y-<A Y, \nabla_{U} Z>A X+ \\
& \quad+<A X, \nabla_{U} Z>A Y \\
& =<\left(\nabla_{U} A\right) Y, Z>A X+<A \nabla_{U} Y, Z>A X+<A Y, \nabla_{U} Z>A X+ \\
& \quad+<A Y, Z>A \nabla_{U} X
\end{aligned}
$$




$$
\begin{gathered}
-<\left(\nabla_{U} A\right) X, Z>A Y-<A \nabla_{U} X, Z>A Y-<A X, \nabla_{U} Z>A Y- \\
-<A X, Z>\left(\nabla_{U} A\right) Y \\
-<A X, Z>A \nabla_{U} Y-<A Y, Z>A \nabla_{U} X+<A \nabla_{U} X, Z>A Y \\
-<A \nabla_{U} Y, Z>A X+<A X, Z>A \nabla_{U} Y-<A Y, \nabla_{U} Z>A X- \\
-<A X, \nabla_{U} Z>A Y=0
\end{gathered}
$$

which shows that $M$ is locally symmetric. In case when $M$ is an anti-invariant submanifold, then (3.15) becomes to

$R(X, Y) Z=\frac{1}{4}(c+1) R^{\prime}(X, Y) Z+<A Y, Z>A X-<A X, Z>A Y$,

where we put $R^{\prime}(X, Y) Z=(X \wedge Y) Z=<Y, Z>X-<X, Z>Y$. It is easily seen that $\nabla_{U} R^{\prime}=0$. Therefore, we see that in this case we have $\nabla_{U} R=0$, completing the proof.

As a corollary of Theorem 3.6 , we have

Theorem 3.8. Let $M$ be a contact normal submanifold of codimension 2 in a Kenmotsu space form $\widetilde{M}(c)$ with $c \neq \neq-1$. If the second fundamental tensor $B$ is parallel or the mean curvature vector field $H$ is parallel or $\nabla_{X} A=0$, then $M$ is an anti-invariant submanifold and the Ricci tensor $S$ and the scalar curvature sare given respectively by

$$
\begin{aligned}
\text { (3.18) } & S(X, Y)=\frac{1}{2}(m-1)(c+1)<X, Y>+(\text { trace } A)<A X, Y>- \\
& <A X, A Y> \\
\text { (3.19) } & s=\frac{1}{2}(m-1)(2 m-1)(c+1)+(\text { trace } A)^{2}-\operatorname{trace} A^{2} .
\end{aligned}
$$
have

Finally, for totally umbilical submanifolds of codimension 2 in $\widetilde{M}(c)$, we

Theorem 3.9. Let $M$ be a proper totally umbilical contact normal submanifold of codimension 2 in a Kenmotsu space form $\widetilde{M}(c)$. Then $M$ is a space of constant curvature $\frac{1}{4}(c+l)$ if dim. $\underline{D}^{2}>I$ and a space of constant curvalure -1 if dim. $\underline{D}^{\perp}=1$.

Proof. We first assume that dim. $\underline{\mathrm{D}}^{\perp}>1$. Then we have $A=0$ and hence $\nabla_{X} A=0$. Thus we see that $M$ is anti-invariant by virtue of Theorem 3.6. Thus from (3.15) we see that $M$ is a space of constant curvature $\frac{1}{4}(c+1)$. Next we assume that dim. $\underline{\mathrm{D}}^{\perp}=1$. Then we see that $\underline{\mathrm{D}}^{\perp}=\{\phi \zeta\}$. Calculating $\widetilde{\nabla}_{x} \phi \zeta$ in 
two ways by using $(1.5),(1.6)$ and $(1.7)$ we have $\nabla_{Y} \phi \zeta+B(X, \phi \zeta)=-\phi A X$. where $X \in D$. Since $M$ is totally umbilical, $B(X, Y)=\langle X, Y\rangle H=\langle X, Y\rangle$ $\left\{-\xi+\frac{1}{2 m-1}\right.$ (irace $\left.\left.A\right) \zeta\right\}$. Putting $Y=\phi \zeta$ in the above equation. we have $B(X, \phi \zeta)=0$ and whence we have $\nabla_{X} \phi \zeta=-\phi A X$. Then we have

$$
\begin{aligned}
& \left(\widetilde{\nabla}_{\Sigma} B\right)(X, \phi \zeta)=\left(\nabla_{\Xi}^{\prime} B\right)(X, \phi \zeta)-B\left(\nabla_{z} X, \phi \zeta\right)-B\left(X, \nabla_{\Sigma} \phi \zeta\right) \\
& =-<\nabla_{-} X, \phi \zeta>-<X, \nabla_{-} \phi \zeta>\xi+\frac{1}{2 m-1}<\nabla_{z} X, \phi \zeta>(\operatorname{trace} A) \zeta \\
& +\frac{1}{2 m-1}<X, \nabla_{-} \phi \zeta>(\operatorname{trace} A) \zeta+\frac{1}{2 m-1}<X, \phi \zeta>(\text { Ztrace } A) \zeta \\
& =0 .
\end{aligned}
$$

Thus, from (3.3), (3.6) with $Z=\phi \zeta$ and taking account of the above identity, we have $\frac{1}{2}(c+1)\langle X, \phi Y\rangle=0$, from which, since $M$ is proper, we see that $M$ is at space of constant curvature-1, completing the proof.

Remark. If $M$ with dim. $\underline{\mathrm{D}}^{\perp}>1$ is in $\bar{M}(-1)$, then we see from (3.15) directly that $M$ is flat. Also, we see that there exist no totally umbilical contact normal submanifolds with $\operatorname{dim} . \underline{D}^{\perp}=1$ in $\widetilde{M}(c)$ with $c \neq-1$.

\section{CONTACT GENERIC NORMAL SUBMANIFOLDS IN KENMOTSU MANIFOLDS}

Let $M$ be a contact generic normal submanifold (not a hypersurface so that dim. $\mathrm{D}^{\perp} \geq 1$, where $\mathrm{D}^{\perp}=\phi T(M)^{\perp}$ ) in a Kenmotsu manifold. We denote by $\underline{\mathrm{D}}$ the orthogonal complementary distribution of $\underline{\mathrm{D}}^{\perp}$ in $T(M)$ so that we may put

(4.1) $T(M)=\underline{\mathrm{D}} \otimes \underline{\mathrm{D}}^{\perp} \quad$ (direct sum).

For a vector field $Y$ tangent to $M$, we put

(4.2) $\phi Y=P Y+F Y$,

where $P Y$ (resp. $F Y$ ) is the tangent (resp. normal) part of $\phi Y$. Then we have
(a) $\langle P Y, X\rangle=-\langle P X, Y\rangle$,
(b) $P^{2} Y=-Y+\phi F Y$.
(c) $F P Y=0$,
(b) $P \phi T(M)^{\perp}=\{0\}$
(e) $P^{3} Y+P Y=0$. 
(c) is obtained by applying $P$ to both handsides of (b) and taking account of (d). Condition (e) shows that the $P$ is an $f$-structure on $M$ (see, [l] or [10]).

The following lemma is valid (see [8]):

Lemma 4.1. Let $M$ be a contact generic normal submanifold of a Kenmotsu manifold $\widetilde{M}$. Then we have

(a) $A_{\phi X} Y=A_{\phi} X$ for $X, Y \in \underline{\mathrm{D}}^{\perp}$, (b) $\underline{\mathrm{D}}^{\perp}$ is always integrable,

(4.4) (c) D is integrable if and only if $B(X, \phi Y)-B(\phi X, Y)=2<\phi X, Y>\xi$,

(d) $M$ is totally umbilical if and only if $B(X, Y)=-\langle X, Y\rangle \xi$ for vector fields $X, Y$ tangent to $M$ provided that dim. $\underline{D}^{+}>I$.

Now we have

Theorem 4.2. If the distribution $D$ is integrable. then the leaves of $\underline{D}$ are totally geodesic in $M$ if and only if

(4.5) $B(X, \phi Y)=<P X, Y>\xi$ for $\forall X, Y \in \underline{D}$.

Proof). For $Z=\phi N\left(N \in T(M)^{\perp}\right)$, we have

$$
\begin{aligned}
& \left\langle\nabla_{X} Y, Z\right\rangle=\left\langle\nabla_{X} Y, \phi N\right\rangle=-\left\langle Y, \widetilde{\nabla}_{X} \phi N\right\rangle=-\left\langle Y,\left(\widetilde{\nabla}_{x} \phi\right) N+\phi \widetilde{\nabla}_{X} N\right\rangle \\
& =-\left\langle Y,\langle\phi X, N\rangle \xi-\eta(N) \phi X-\phi A_{N} X+\phi \nabla_{X}^{\perp} N\right\rangle \\
& =\langle Y, \phi X\rangle \eta(N)+\left\langle Y, P A_{N} X\right\rangle-\left\langle Y, \phi \nabla_{X}^{+} N\right\rangle \\
& =\langle Y, P X>\eta(N)-<B(P Y, X), N\rangle \\
& =\langle<Y, P X>\xi, N>-\langle B(P Y, X), N\rangle \\
& =\langle\langle Y, P X\rangle \xi-B(P Y, X), N\rangle \text {. }
\end{aligned}
$$

from which we see that the leaves of $\underline{\underline{D}}$ are totally geodesic in $M$ if and only if (4.5) holds.

Theorem 4.3. The leaves of the distribution $D^{\perp}$ are totally geodesic in $M$ if and only: if

(4.6) $<B\left(\underline{D}, \underline{\mathrm{D}}^{\perp}\right), \phi \underline{\mathrm{D}}^{\perp}>=\{0\}$. 
Proof. Let $Z=\phi N, W=\phi N^{\prime}\left(N, N^{\prime} \in T(M)^{\mu}\right)$ and $X \in \underline{\mathrm{D}}$. Denote by $B^{\prime}$ the second fundamental form of a maximal integral submanifold $M^{\perp}$ of $\underline{D}^{\perp}$ in $M$. Then we have

$$
\begin{aligned}
&\langle B(Z, X), \phi W\rangle=\left\langle\nabla_{7} X, \phi W\right\rangle=-\left\langle X, \widetilde{\nabla}_{Z} \phi W\right\rangle= \\
&=-\left\langle X,\left(\widetilde{\nabla}_{Z} \phi\right) W+\phi \widetilde{\nabla}_{Z} W\right\rangle \\
&=-\left\langle X,\langle\phi Z, W\rangle \xi-\eta(W) \phi Z+\phi \nabla_{Z} W+\phi B(Z, W)>\right. \\
&=-\left\langle X, \phi \nabla_{Z} W\right\rangle=\left\langle\phi X, \nabla_{Z} W\right\rangle=\left\langle\phi X, B^{\prime}(Z, W\rangle,\right.
\end{aligned}
$$

from which we see that the leaves of $\underline{D}^{\perp}$ are totally geodesic in $M$ if and only if (4.6) holds.

We now put $\left(\bar{\nabla}_{X} P\right) Y=\nabla_{X} P Y-P \nabla_{X} Y$ and $\left(\hat{\nabla}_{X} F\right) Y=\nabla_{X}^{\perp} F Y-F \nabla_{X} Y$. We call $P$ (resp. $F$ ) is parallel, if $\left(\bar{\nabla}_{X} P\right) Y=0$ (resp. $\left(\bar{\nabla}_{X} F\right) Y=0$ ). We have

Lemma 4.4. The following relations hold:

$$
\begin{aligned}
& (4.7) \quad\left(\hat{\nabla}_{X} P\right) Y=A_{\digamma Y} X+\phi B(X, Y), \\
& (4.8) \quad\left(\hat{\nabla}_{X} F\right) Y=-B(X, P Y)+<P X, Y>\xi .
\end{aligned}
$$

Proof. We have

$$
\begin{aligned}
\widetilde{\nabla}_{X} \phi Y & =\widetilde{\nabla}_{X} P Y+\widetilde{\nabla}_{X} F Y=\nabla_{X} P Y+B(X, P Y)-A_{F Y} X+\nabla_{X}^{\perp} F Y \\
& =\left(\hat{\nabla}_{X} P\right) Y+P \nabla_{X} Y+B(X, P Y)-A_{F} X+\left(\hat{\nabla}_{X} P\right) Y+F \nabla_{X} Y .
\end{aligned}
$$

On the other hand, using (1.5), we have

$$
\begin{aligned}
\widetilde{\nabla}_{X} \phi Y & =\left(\widetilde{\nabla}_{X} \phi\right) Y+\phi \widetilde{\nabla}_{X} Y=\left\langle\phi X, Y>\xi-\eta(Y) \phi X+\phi \nabla_{X} Y+\phi B(X, Y)\right. \\
& =<P X, Y>\xi+P \nabla_{X} Y+F \nabla_{X} Y+\phi B(X, Y) .
\end{aligned}
$$

Therefore, comparing the above two equations and taking the tangent and normal parts, we have (4.7) and (4.8) respectively.

Proposition 4.5. If $F$ is parallel, then the distribution $D$ is integrable and its leaves are totally geodesic in $M$.

Proof. Since $F$ is parallel, from (4.8), we have $B(X, P Y)=\langle P X, Y\rangle \xi$ for $\forall X, Y \in \underline{\mathrm{D}}$ and hence we have $B(X, P Y)-B(Y, P X)=\langle P X, Y\rangle \xi-$ 
$\langle P Y, X>\xi=2<P X, Y>\xi$, which shows that $\mathrm{D}$ is integrable and its leaves are totally geodesic in $M$ by virtue of (c) of (4.4) and Theorem 4.2, which completes the proof.

Lemma 4.6. The f-structure $P$ is parallel if and only if

$$
<B(\underline{\mathrm{D}}, \underline{\mathrm{D}}), \phi \underline{\mathrm{D}}^{-}>=\{0\} \underline{\text { and }}<B\left(\underline{\mathrm{D}}, \underline{\left.\mathrm{D}^{-}\right)}, \phi \underline{\mathrm{D}}^{\perp}>=\{0\} .\right.
$$

Proof. For a tangent vector field $U$ to $M$, making an inner product from this with (4.7), we have

$$
<\left(\hat{\nabla}_{X} P\right) Y, U>=\left\langle A_{F Y} X, U>-<B(X, Y), \phi U>.\right.
$$

Thus, if we put $U=W \in \underline{\mathrm{D}}^{\perp}$, then we have

$$
\begin{gathered}
\left\langle\left(\hat{\nabla}_{X} P\right) Y, W>=\left\langle A_{F Y} X, W>-\langle B(X, Y), \phi W\rangle .\right.\right. \\
=\left\langle A_{F Y} W, X>-\left\langle A_{F W} Y, X>=\left\langle A_{F Y} W-A_{F W} Y, X>=0\right.\right.\right.
\end{gathered}
$$

by virtue of (a) of (4.4). And, if we put $U=V \in \underline{\mathrm{D}}$, then we have

$$
\begin{gathered}
\left\langle\left(\tilde{\nabla}_{X} P\right) Y, V\right\rangle=\left\langle A_{F,} X, V\right\rangle-\langle B(X, Y), \phi V\rangle . \\
=\left\langle A_{F}, X, V\right\rangle-\langle B(X, V), F Y\rangle=\langle B(X, V), \phi Y\rangle .
\end{gathered}
$$

Since $X$ is a tangent vector field to $M$, we see that $P$ is parallel if and only if (4.9) holds, which completes the proof.

Corollary 4.7. If the f-structure $P$ is parallel, then the leaves of $\underline{D}^{\perp}$ are totally geodesic in $M$.

Corollary 4.8. If both $P$ and F are parallel, then $D$ is integrable and the leaves of both $\underline{D}$ and $\underline{D}^{\perp}$ are torally geodesic in $M$.

Definition. $M$ is called to be a contact generic normal product if $D$ is integrable and $M$ is locally a Riemannian product of an invariant submanifold $M^{\top}$ and an anti-invariant submanifold $M^{\perp}$, where $M^{\top}$ (resp. $M^{\perp}$ ) is a leaf of $\underline{D}$ (resp. $\left.\underline{D}^{\perp}\right)$. 
Theorem 4.9. The following statements are mutually equivalents.

(a) $\mathrm{M}$ is a contact generic normal product.
(b) $B(X, \phi Y)=\left\langle\phi X, Y>\xi\right.$ and $A_{F Z} Y=0$ for $\forall X, Y \in \underline{\mathrm{D}}$ and $Z \in \underline{\mathrm{D}}^{\perp}$.
(c) $B(X, \phi Y)=<\phi X, Y>\xi$ and $B(X, Z)=0$ for $\forall X, Y \in \underline{\mathrm{D}}$ and $Z \in \underline{\mathrm{D}}^{\perp}$.

Proof. Assume that (a) holds. Then we have

$$
B(X, \phi Y)-B(\phi X, Y)=<\phi X, Y>\xi-<\phi Y, X>\xi=2<\phi X, Y>\xi
$$

from which we see that $\underline{D}$ is integrable and its leaves are totally geodesic in $M$ by virtue of (c) of (4.4) and Theorem 4.2. Moreover, making an inner product with $W \in \underline{D}-$ in $A_{Y: \zeta} Y=0$, we have

$$
0=\left\langle A_{F Z} Y, W\right\rangle=\langle B(Y, W), F Z\rangle=\langle B(Y, W), \phi Z\rangle
$$

that is, $\angle B\left(\underline{D}, D^{+}\right), \phi D^{\perp}>=\{0\}$, which shows that the leaves of $\underline{D}^{\perp}$ are totally geodesic in $M$ by virtue of ']heorem 4.3. Thus $M$ is a contact generic normal product. Conversely, assume that $M$ is a contact generic normal product. Then we have $\nabla_{U} X \in \mathrm{D}$ and $\nabla_{U} Z \in \underline{\mathrm{D}}^{\perp}$ for $X \in \underline{\mathrm{D}}, Z \in \underline{\mathrm{D}}^{\perp}$ and $U \in T(M)$. Putting $Y=Z \in \underline{\mathrm{D}}^{-}$and $X=U$ in (4.7), we have

$$
\left.\hat{\nabla}_{U} P\right) Z=\nabla_{U} P Z-P \nabla_{U} Z=A_{F Z} U+\phi B(U, Z)
$$

from which we have

$$
A_{F \%} U=-\phi B(U, Z)
$$

because of $P Z=0$ and $\nabla_{j} Z \in \underline{D}^{\perp}$. Then, making an inner product from this with $\phi Y(Y \in \underline{\mathrm{D}})$, we have

$\left\langle A_{F Z} U, \phi Y>=-\langle\phi B(U, Z), \phi Y>=-\langle B(U, Z), Y>+\eta(B(U, Z)) \eta(Y)\right.$ $=0$.

Putting $Z=\phi N\left(N \in T(M)^{\perp}\right)$ in the above equation, we have

$$
\begin{aligned}
0= & <A_{\Gamma,} U, \phi Y>=<B(U, \phi Y), F Z>=<B(U, \phi Y), \phi^{2} N>= \\
& <B(U, \phi Y),-N+\eta(N) \xi> \\
=- & <B(U, \phi Y), N>+\eta(N)<B(U, \phi Y), \xi> \\
=- & <B(U, \phi Y), N>-\eta(N)<U, \phi Y>
\end{aligned}
$$




$$
\begin{aligned}
& =-\langle B(U, \phi Y), N\rangle-\langle\xi, N\rangle\langle U . \phi Y\rangle \\
& =-\langle B(U, \phi Y)+\langle U, \phi Y>\xi, N\rangle .
\end{aligned}
$$

from which we have

(**) $\quad B(U, \phi Y)=-\langle U, \phi Y>\xi=\langle\phi U, Y>\xi$.

Setting $U=X \in \underline{\mathrm{D}}$ in the above equation, we have $B(X, \phi Y)=\langle\phi X, Y\rangle \xi$. Replacing $Y$ by $\bar{\phi} Y$ in this equation, we have $B(X, Y)=\langle X, Y\rangle \xi$. Thus we have.

$(* * *)<A, X X, Y\rangle=\langle B(X, Y), F Z\rangle=\langle B(X, Y), \phi Z\rangle=$ $=\langle<X, Y>\xi, \phi Z\rangle=\langle X, Y\rangle\langle\xi, \phi Z\rangle=0$.

Also, setting $U=Z \in \underline{\mathrm{D}}^{*}$ in $(* *)$, we have $B(Z, \phi Y)=0$. Then, replacing $Y$ by $\phi Y$ in this equation, we have $B(Z, Y)=0$. Therefore, for $W \in D^{\perp}$, we have $\left\langle A_{\mu,} Y, W\right\rangle=\left\langle Y, A_{F,} W\right\rangle=\left\langle Y, A_{F W} Z\right\rangle=\langle B(Y, Z), F W\rangle=0$. This, together with ( $\left.{ }^{* * *}\right)$, implies $A_{F: Y} Y=0$. Finally, to show $A_{F Z} X=0$ $\Longleftrightarrow B(X, Z)=0$, we look back at $\left(^{*}\right)$ and put $U=X \in \underline{D}$ Then we have $0=A, Z X=-\phi B(X, Z)$. Applying $\phi$ to $0=\phi B(X, Z)$, we have

$$
\begin{aligned}
0 & =\phi^{2} B(X, Z)=-B(X, Z)+\eta(B(X, Z)) \xi=-B(X, Z)+<B(X, Z), \xi>\xi \\
& =-B(X, Z)+<X, Z>\xi=-B(X, Z) .
\end{aligned}
$$

Thus, (a) $\Leftrightarrow$ (b) is established, which completes the proof.

Remark. A contact generic normal submanifold with parallel $\mathrm{P}$ and $\mathrm{F}$ is a contact generic normal product (Cor. 4.8).

Proposition 4.10. A tolally umbilical proper contact generic normal submanifold in a Kenmotsu manifold is a contact generic normal product if dim. $\underline{D} \rightarrow 1$.

Proof. Since $M$ is totally umbilical, we have $B(X, Y)=-\langle X, Y\rangle \xi$. Then we have $B(X, \phi Y)=-<X, \phi Y>\xi=\langle\phi X, Y>\xi$. Moreover, for a tangent vector field $U$ to $M$ we have

$$
\begin{aligned}
\left\langle A_{1} \%, U, U>=\right. & <B(X, U), F Z>=<-<X, U>\xi, \phi Z>- \\
& -<X, V><\xi, \phi Z>=0 .
\end{aligned}
$$

Whence, by Theorem 4.9, we see that $M$ is a contact generic normal product. 
Theorem 4.11. Let $M$ be a proper contact generic normal product in a Kenmotsu space form $\widetilde{M}(c)$. Then $c=-1$. and

(4.11) $\langle R(X, Y) Z, W\rangle=-(\langle Y, Z\rangle\langle X, W\rangle-\langle X, Z\rangle\langle Y, W\rangle)+$ $+<B(X, W), B(Y, Z)>$ $-<B(Y, W), B(X, Z)\rangle$

(4.12) $S(X, Y)=-(n-1)<X, Y\rangle+n\langle B(X, Y), H\rangle-$ $\sum_{i=1}^{n}\left\langle B\left(X, e_{i}\right), B\left(Y, e_{i}\right)\right\rangle$,

(4.13) $s=-(n-1) n+n^{2}<H, H>-\sum_{j=1}^{n}\left\|B\left(e_{i}, e_{j}\right)\right\|^{2}$,

where $\left\{e_{i}\right\}$ is an orthonormal basis of $T(M)$

Proof. Since $M$ is a contact generic normal product we have $\left(\bar{\nabla}_{X} B\right)$ $(Y, Z)=0$ for $X, Y \in \underline{D}$ and $Z \in \underline{D}^{-}$by virtue of Theorem 4.9. Therefore, (2.21) reduces to $\frac{1}{2}(c+1)<X, P Y>F Z=0$. Replacing $Y$ by $P X$ in the above equation, we have $(c+1)\|X\|^{2} F Z=0$, from which, since $M$ is proper, we have $c=-1$. Then $(4.11)-(4.13)$ follow from $(1.16)$ with $c=-1$, completing the proof.

As for the normal connection of $M$. we have

Proposition 4.12. Let $M$ be a contact generic normal product in a Kenmotsu space form $\widetilde{M}(c)$. Then $c=-I$ and the normal connection of $M$ is flat if and only if Weingarten maps are commutative, i.e., $\left[A_{N}, A_{N}\right]=0$.

Proof. This follows from Theorem 4.9 and (1.18).

Theorem 4.13. Let $M$ be a totally umbilical proper comtact generic normal product with dim. $\underline{D}^{\prime}>I$ in a Kenmotsu space form $\widetilde{M}(c)$. Then $M$ is flat.

Proof. By assumption, we have $B(X, Y)=-\langle X, Y\rangle \xi$, so that substituting this into (4.11) of Theorem 4.11 , we have $R=0$, which means that $M$ is flat. 
Hereafter let $M$ be an orientable hypersurface in a Kenmotsu manifold $\widetilde{M}$ whose structure vector field $\xi$ is normal to $M$. It is easily see that $H=-\xi$, because $M$ is a hypersurface. First, we have

Theorem 4.14. Let $M$ be a hvpersurface in a Kenmotsu manifold $\widetilde{M}$ whose structure vector field $\xi$ es normal to $M$. Then $M$ is an extrinsic sphere. that is, $M$ is totally umbilical and the mean curvature vector field $H$ is parallel.

Proof. Differentiating $H=-\xi$ covariantly with respect to a tangent vector field $X$, we have

$$
\widetilde{\nabla}_{\lambda} H=-A_{H} X+\nabla_{\tilde{X}} H=-\widetilde{\nabla}_{\lambda} \xi=A_{\xi} X-\nabla_{\dot{H}} \xi,
$$

from which, comparing the tangent and normal parts and taking account of $A_{\xi} X=-X$ and $\nabla_{\bar{Y}} \xi=0$, we have $A_{H} X=X$ and $\nabla_{\bar{X}} H=0$. Thus, $M$ is an extrinsic sphere, completing the proof.

Theorem 4.15. Let $M$ be a hypersurface in a Kenmotsu space form $\tilde{M}$ (c) whose structure vector field $\xi$ is normal to $M$. Then we have

$$
\begin{aligned}
& \text { (4.14) } \quad R(X, Y) Z=\frac{1}{4}(c+1) \mid<Y, Z>X-<X, Z>Y+<X, \phi Z>\phi Y- \\
& -<Y, \phi Z>\phi X+2<X, \phi Y>\phi Z\}, \\
& \text { (4.15) } S(X, Y)=\frac{1}{2}(c+1)(m+1)<X, Y>, \\
& \text { (4.16) } s=(c+1) m(m+1),
\end{aligned}
$$

Proof. Since $M$ is totally umbilical by Theorem 4.14, substituting $B(X, Y)=\langle X, Y\rangle H$ into (1.16) and taking account of $\langle H, H\rangle=1$, we obtain (4.14) directly.

Theorem 4.16. Let $M$ be a hypersurface in a Kenmotsu space form $\widetilde{M}(c)$ whose structure vector field $\xi$ is normal to $M$. Then $M$ is locally symetric.

Proof. Since $\left(\nabla_{W}, R\right)(X, Y) Z=\nabla_{\mathcal{W}^{\prime}} R(X, Y) Z-R\left(\nabla_{\mathbb{U}} X, Y\right) Z-$ $R\left(X, \nabla_{H^{\prime}} Y\right) Z-R(X, Y) \nabla_{H^{\prime}} Z$, by direct calculations, we have 


$$
\begin{gathered}
\left(\nabla_{W}, R\right)(X, Y) Z=\frac{1}{4}(c+1)\left\{<X,\left(\hat{\nabla}_{W} P\right) Z>P Y-<Y,\left(\bar{\nabla}_{W} P\right) Z>P X+\right. \\
+<X, P Z>\left(\hat{\nabla}_{W}, P\right) Y \\
\left.-<Y, P Z>\left(\hat{\nabla}_{W} P\right) X+2<X,\left(\hat{\nabla}_{W} P\right) Y>P Z+2<X, P Y>\left(\hat{\nabla}_{W} P\right) Z\right\} \\
=\frac{1}{4}(c+1)\{<X, \phi B(W, Z)>P Y-<Y, \phi B(W, Z)>P X \\
+<X, P Z>\phi B(W, Y)-<Y, P Z>\phi B(W, X) \\
+2<X, \phi B(W, Y)>P Z+2<X, P Y>\phi B(W, Z)\} \\
=0 \text { (since } \phi B(X, Y)=0, \text { etc. }),
\end{gathered}
$$

which shows that $M$ is locally symmetric, completing the proof.

Theorem 4.17. Let $M$ be a hypersurface in a Kenmotsu space form $\widetilde{M}$ (c) whose structure vector field $\xi$ is normal to $M$. Then $M$ is flat if and only if $i=-I$ or, equivalently, the scalar sof $M$ is 0 .

Proof. If $c=-1$, then we have $R=0$ by (4.15). Conversely, we assume that $M$ is flat, $R=0$. Then, putting $Z=X$ and $Y=\phi X$ in (4.15), we have

$$
\begin{aligned}
0= & \frac{1}{4}(c+1)\left\{<\phi X, X>X-<X, X>\phi X+<X, \phi X>\phi^{2} X-\right. \\
& \left.\quad<\phi X, \phi X>\phi X+2<X, \phi^{2} X>\phi X\right\} \\
= & -(c+1)\|X\|^{2} \phi X .
\end{aligned}
$$

from which we have $c=-1$. And, $c=-1 \Leftrightarrow s=0$ is trivial by (4.16), completing the prool.

Corollary 4.18. A hypersurface $M$ in a Kenmotsu space form $\tilde{M}(c)$ with $c \neq-1$ whose structure vector field $\xi$ is normal to $M$ is Einsteinian.

Proof. This follows from (4.15). 


\section{References}

[1] A. BEsAnCU, Geomerry of CR submanifolds, Mathematics and Its applications, D. Reidel Publishing Company, 1986.

[2] D. JANSEN and 1.. VANHI:CKE. Almost contact structure and curvature tensors, Kodai Math. J.. 4(1981) 1-27.

[3] K. Kenmotsu, A class of almost contact Riemannian manifolds, Tohoku Math. J., 24 ( I972) 93-103.

[4] M. KOBAYASti, Contact generic submanifolds of a certain class of almost comact manifolds, to appear in Tensor.

[5] - Semi-invariant submanifolds in an f-manifold with complemented frames, to appear in Tensor.

[6] -. Simmetic twofold $C R$ stubmanifolds in a Euclidean space $R^{4 m}$. Coll. Math. 57(1987) 231-240.

[7] -, Compact orientable submanifolds with parallel mean curvature and with flat normal connedion in a 5 -manifold of constant f-sectional curvature. submitted to Proc. Amer. Math. Soc.

[8] N. PAPAGHIUC. Semi-invariant submanifolds in a Kenmotsu manifold. Ren. Math., 3(1983) 607-622.

[9] S. SASAKi, Almost conact manifolds, Lecture notes, Tohoku Univ., 1965.

[10] K. YANO and M. KON, CR Submanifolds of Kaehlerian and Sasakian manifolds, Birkhauser, Boston, 1983.

Departmetr of Mathemitics

Iowal University

Sakudo, Saltama

Recibido: 7 de junio de 1940

$\mathrm{J} \triangle \mathrm{P} \wedge \mathrm{N}$ 